\title{
International viewpoint and news
}

\section{Creating a European portal for mapping geohazards \\ Written by Anne Marie de Grosbois}

On March 1, 2011, the project entitled "PanGeo-Enabling Access to Geological Information in Support of Global Monitoring for Environment \& Security" was launched. This new European initiative is working towards providing online information on geological hazards in the urban areas of the European Union. Although its main users will likely be decision makers and planning authorities, the general public also will have free access to this. The 3 -year project is financed by the EU and involves a consortium of private companies and universities that specialize in satellite InSAR processing, novel visualisations, INSPIRE-compliant webmap serving, GIS and validation. The project also involves 27 European Geological Surveys.

Its UK project coordinator Fugro NPA Ltd, a world leading satellite mapping specialist, points out that as a user makes an online enquiry, "the PanGeo web-portal will automatically integrate the geohazard data with the Urban Atlas to highlight the landcover polygons influenced. Mousing over polygons will hyperlink to interpretative text."

The geological hazards will be analyzed for fifty-two European towns and cities with the highest population density. The EuroGeoSurveys reported that the accessed information "will be obtained by integrating terrainmotion data from a satellite technology called PSI (Persistent Scatterer InSAR) with existing, known information on geohazards already held by the National Geological Surveys, and the exposure data inherent within the EU's Urban Atlas (landcover/use of European urban areas)."

The project also has a group in place that will provide feedback and have the task of testing the project activities in order to verify the effectiveness of the results and information accessed at the portal.

A. M. de Grosbois ( $\square)$

Friedmar-Brendel-Weg 9a, 09599 Freiberg, Germany

e-mail: degrosbois@blue-cable.de 